Тараненко М. Е.

\title{
СОВРЕМЕННЫЙ ПОДХОД К ОЦЕНКЕ ТЕХНОЛОГИИ ОБРАБОТКИ ЛИСТОВЫХ ДЕТАЛЕЙ ДАВЛЕНИЕМ
}

В современной конкурентной борьбе за рынки сбыта выпускаемой продукции выигрывает тот производитель, качество продукции которого выше. Для объективной оценки качества используют методы теоретической квалиметрии - науки о количественной оценке качества. Известны технические приложения таких методов при изготовлении сортового проката [1], агроквалиметрии [2], квалиметрии в транспорте [3], в листовой штамповке [4]. В НАКУ «ХАИ» проводятся исследования использования методов квалиметрии в приложении к технологии производства летательных аппаратов, в частности, к штамповке крупногабаритных листовых деталей (КЛД) разными методами. Для таких деталей использование квалиметрического подхода к основным этапам первой части жизненного цикла продукции особо важно, т. к. при подготовке производства и их производстве необходимы большие сроки и затраты на изготовление всего технологического оснащения, большие размеры заготовок и достаточно длительный процесс изготовления. Часто такие детали нужны в приоритетном или малосерийном производстве и недоработки в проектировании деталей и техпроцессах увеличивают их себестоимость.

Целью работы является установление общих подходов к квалиметрической (комплексной) оценке качества наиболее сложных и дорогостоящих листовых деталей, во многом определяющих эффективность производства всего изделия.

В общем случае квалиметрический подход к организации производства продукции требует рассмотрения трех ее видов: деталей, машин и технологических процессов. Но в данном случае можно ограничиться рассмотрением только двух видов деталей и процессов.

Количество деталей определяется присущими им многочисленными свойствами. В данном рассмотрении можно ограничиваться несколькими. В свою очередь свойства оценивают соответствующими показателями качества (ПК).

Свойства крупногабаритных листовых деталей (КЛД). Из большого количества свойств, присущих расходуемой продукции, для названных деталей более актуальным для квалиметрического анализа являются следующие группы свойств:

- функционального назначения;

- технологичности;

- воспринятой технологической наследственности (ТН);

- экономические.

Показатели назначения - одна из важнейших групп ПК, характеризующих назначение, область применения, транспортабельность, конструктивные и другие особенности изделия. Они применимы для всех видов деталей вне зависимости от цели оценки уровня качества и условий ее применения.

Функции, выполняемые крупногабаритными листовыми деталями, можно отнести к трем видам:

- функции образа некоторого объема по заданной форме (аэродинамические обшивки, параболические зеркала). К ним предъявляются высокие (очень высокие) требования по точности формы;

- функции передачи распределенных механических нагрузок (нервюры, диафрагмы). Они требуют заданной точности формы, повышенной точности размеров и минимальных значений вредных остаточных напряжений;

- функции ограничения некоторых объемов (стенки сосудов, днища, детали интерьеров транспортных средств). Для них присущи нормальные или пониженные требования по точности и нормальные требования по внешнему виду. 
Показатели технологичности конструкции детали - важная составная часть оценки качества изделия, поскольку в комплексе они характеризуют эффективность конструктивнотехнологических решений с точки зрения приспособленности (адаптивности) изделия для изготовления на конкретном производстве с заданными условиями выпуска и минимальной себестоимостью. Они должны задаваться на этапе проектирования или при технологической проработке изделия. Их состав для детали ОМД рассмотрен далее.

Показатели ТН - достаточно важны для оценки качества готовых изделий, так как могут существенно влиять на функции назначения и содержание маршрута изготовления изделия в целом. Например, коробление листовых деталей затрудняет автоматизацию процесса сборки.

Показатели технологичности листовых деталей. Технологичность - свойство детали, конструкции, машины, дающие возможность ее изготовления на данном производстве в заданные сроки, в количестве и с указанным качеством при минимальных затратах. Естественно, на разных предприятиях и при различных условиях показатели технологичности могут отличаться. Это свойство комплексное и может быть расчленено на ряд более простых свойств (рис. 1).

Это определение несколько отличается от записанных ранее в нормативных документах по форме, но по смыслу соответствует им. Нами предложен состав показателей свойств технологичности.

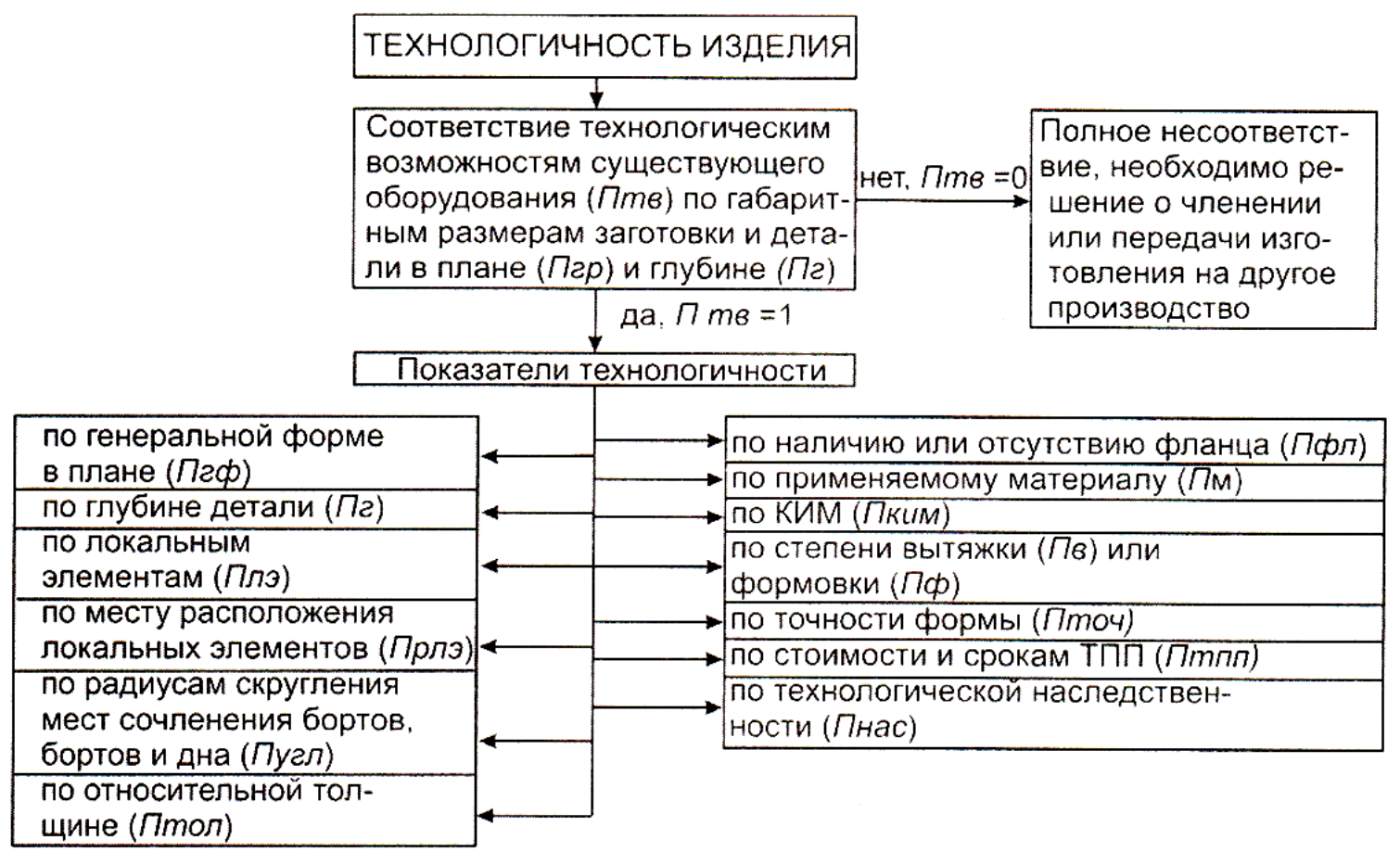

Рис. 1. Структура показателей технологичности

Показатели технологичности являются важной составной частью оценки качества изделий, поскольку комплексно характеризуют конструкцию с точки зрения приспособленности к данному производству. Поэтому проработка изделия на технологичность является обязательным этапом при подготовке производства.

Алгоритм определения комплексного показателя технологичности начинается с оценки соответствия габаритных размеров листовой заготовки и детали технологическим возможностям оборудования $\left(\Pi_{m в}\right)$. Он может принимать два значения: 1 - соответствия техническим возможностям и 0 - полное несоответствие. Последнее влечет за собой принятие соответствующих управленческих решений.

Далее приведены показатели технологичности по другим свойствам. 
Большинство из этих свойств, характеризуемых определенным показателем качества, являются комплексными, включающими в себя или обеспечиваемые более простыми свойствами. Количественно оценить уровень этих свойств или установить численные значения частных показателей качества (ПК) является одной из задач технологической квалиметрии. Например, рассмотрим свойство технологичности КЛД. Оно определяется следующими факторами:

- габаритными размерами в плане, глубиной детали, а также их соответствием технологическим возможностям оборудования; жения;

- генеральной формой детали, формой ее локальных элементов и местом их располо-

- толщиной, допустимой разнотолщинностью и местом ее расположения;

- конструктивными элементами детали (размеры, углы гиба, относительная толщина, коэффициенты вытяжки);

- точностью тех или иных поверхностей или их взаимным расположением;

- стоимостью и длительностью технологической подготовки производства;

- коэффициентом использования материала по листу или рулону;

- параметрами технологической наследственности.

Строго говоря, некоторые из приведенных ниже показателей являются комплексными, например, показатель технологичности по точности можно разделить на ряд более частных показателей:

- точности разных размеров детали;

- точности вогнутых или выпуклых поверхностей;

- точности толщины детали;

- точности по двум координатам зон сопряжения поверхностей.

Показатель технологичности по локальным элементам может состоять из следующих показателей:

- наличия локальных элементов;

- места их расположения (борт или стенка);

- направленности выпуклости локальных элементов в направлении действия нагрузки или наоборот и др.

Эти факторы (при известных объемах производства) определяют выбор схемы и метода штамповки, необходимое оборудование, а, следовательно, себестоимость и трудоемкость изготовления деталей.

Для определения комплексного квалиметрического показателя технологичности предлагается использовать следующую зависимость:

$$
\Pi^{\text {техн }}=\frac{1}{n} \sum_{i=1}^{n} \Pi_{i}
$$

где: $\Pi_{i}$ - показатели технологичности по отдельным составляющим ее фактором; $n$ - количество учитываемых факторов.

Здесь не учитывается весомость каждого показателя, так как трудно определить влияние ее учета на адекватность полученного результата.

Наиболее трудно определить численные значения показателя генеральной формы детали $\Pi_{2 \phi}$. Здесь нами предположено, что любую крупногабаритную деталь можно представить в виде оболочки определенной генеральной формы и наложенных на неё локальных элементов с поперечными размерами, значительно меньшими размеров генеральной формы. Всё разнообразие форм можно расположить вдоль линеек шкал, соответствующих соотношению площадей, соотношению сторон и степени неплоскостности поверхности матрицы, на которую укладывается заготовка (рис. 2). Последовательность их расположения соответ- 
ствует сложности формы. По горизонтальной оси формы: круглая, многоугольная, квадратная, треугольная, полость с выпукло-вогнутыми участками, полость сложной формы. По вертикальной оси - полутор, спиральный полутор. По оси изометрии - эллипсы (овалы) с разным соотношением сторон, желоба. Цифрами показаны значения соотношения площадей фигуры и вписанной окружности

Влияние фактора формы полости в плане на сложность и трудоёмкость штамповки достаточно сложно оценить каким-либо функционально-объективным показателем. На основании опыта штамповки сложных деталей был предложен следующий подход - оценить форму полости матрицы отношением её площади в плане к площади вписанной окружности. Примеры этих значений показаны на рис. 2. А затем проверить отношение этих показателей результатам опытной штамповки круглого днища средней эллиптичности, квадратной коробчатой детали и детали сложной формы. Такое отношение равно 1,0 : 1,48 : 2,42. Что в первом приближении соответствует значениям, полученным по отношению площадей $1,0: 1,27:(1,88 \ldots 4,0)$. Необходимо уточнить, что это отношение получено при анализе процесса штамповки-вытяжки деталей жидкой или эластичной средой и учитывает трудоёмкость изготовления оснастки. Расхождение значений объясняется неполным соответствием форм детали, но может быть уточнено с увеличением опытных статистических данных.

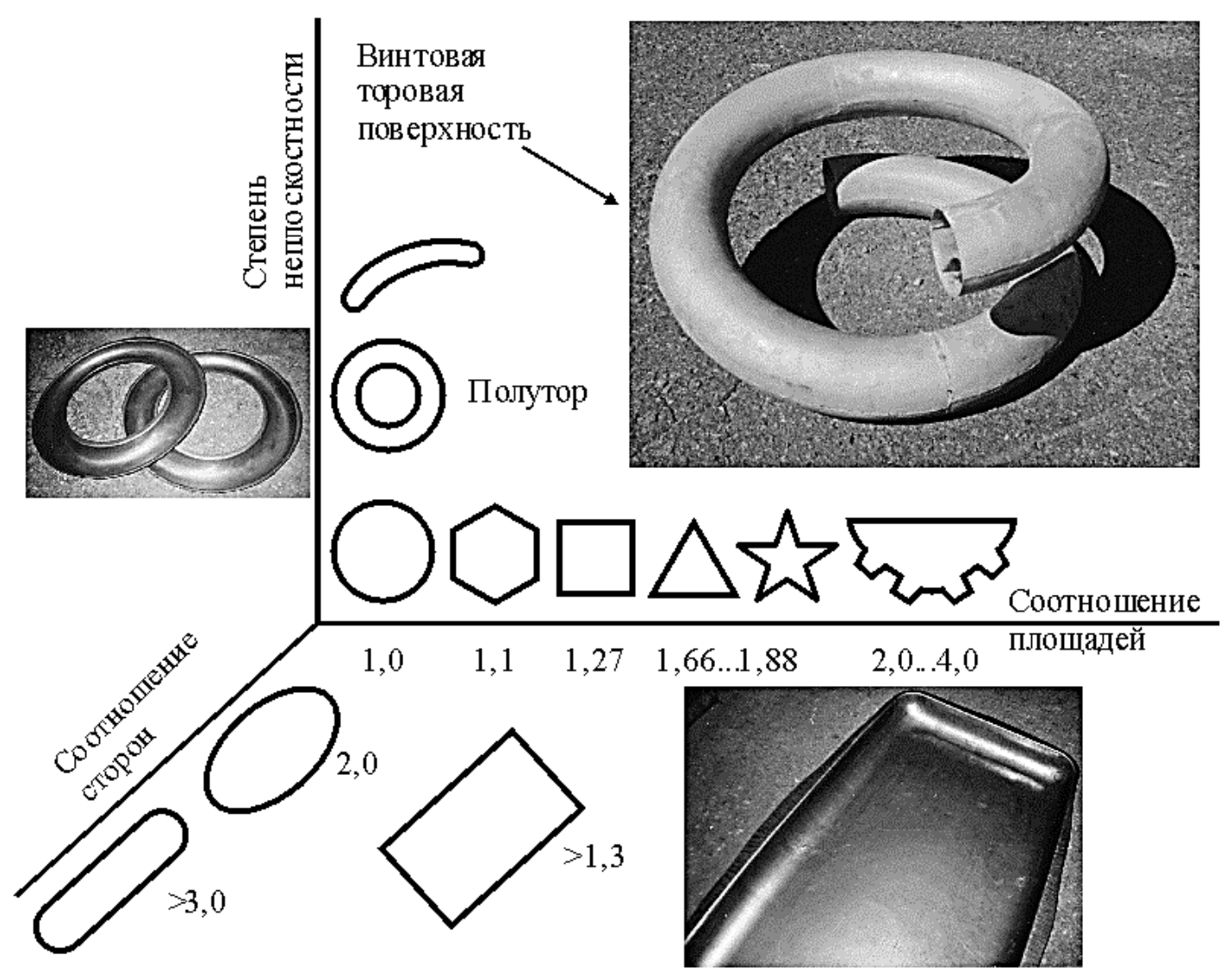

Рис. 2. Влияние формы полости в плане на сложность процесса штамповки-вытяжки листовых деталей.

Для суммирования показателей формы деталей, содержащих все элементы сложности формы используются методы векторного анализа.

Такой подход дает возможность получения численного значения показателя технологичности сложности формы $\Pi_{\phi}^{\text {mехн }}$.

Влияние глубины детали на затрачиваемую для штамповки энергию, а следовательно, на показатель технологичности по глубине оценивается в работе [5]. Там сделан вывод о том, 
что частный показатель технологичности по потребленной для основных штампуемых форм деталей $\Pi_{н}^{\text {mехн }}$ увеличивается пропорционально второй степени глубины детали $H$. С учетом стоимости изготовления оснастки он записывается в виде:

$$
\Pi_{{ }_{H}}^{\text {mexH }}=f_{1}\left(H^{2}\right)+f_{2}\left(H^{2,5 \ldots, 4,0}\right) .
$$

Диапазон степени $H$ определяется методом изготовления оснастки.

Такая функция достаточно резко возрастает с увеличением параметра $H$, что позволяет сделать вывод о превалирующем влиянии глубины детали на показатель её технологичности.

Влияние величины радиусов сопряжения бортов между собой и стенки детали с бортами на соответствующий частный показатель технологичности предлагается оценивать по сложности и количеству необходимых технологических приёмов, используемых при штамповке коробчатых деталей. Количественным показателем сложности формообразования трёхгранного угла в этом случае служит местный коэффициент вытяжки. Можно показать, что значения этого параметра сопоставимы со шкалой сложности технологических приёмов, приведенной в работе [6]. Эти численные значения получены при штамповке коробок эластичной и жидкой средой. Частным показателем технологичности угловых локальных элементов является П (рис. 3).

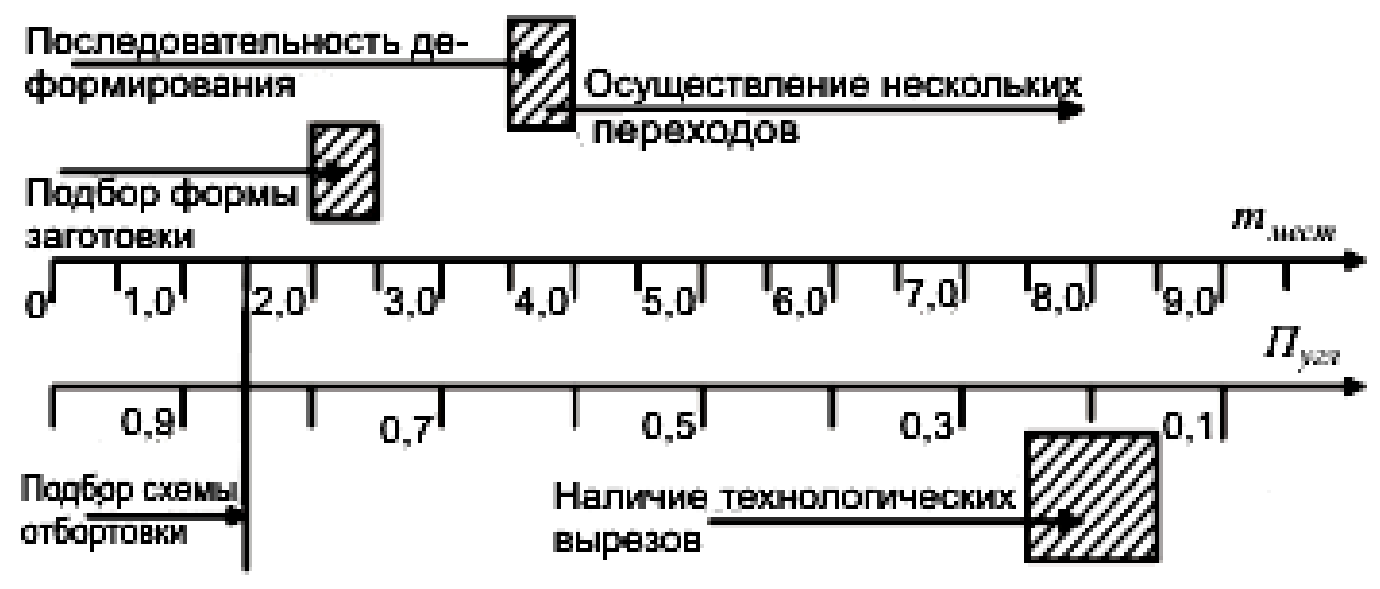

Рис. 3. Условное совмещение шкалы местного коэффициента вытяжки в угловой зоне

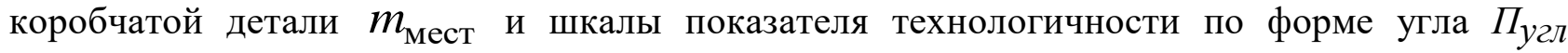
(цифры шкалы - условные)

Подобным опытно-статическим подходом можно установить частные показатели технологичности по точности, длительности и стоимости ТПП и другим факторам, определяющим комплексный показатель технологичности при сопоставимости используемых шкал.

При выборе технологии штамповки КЛД необходимо учитывать современный функционально-ориентированный подход к определению преимуществ выбираемых вариантов [7]. Он заключается в необходимости согласования требуемого уровня технологических воздействий технологически строго соответствующим зонам детали.

Большинство КЛД имеют участки, требующие для своего формообразования потоков энергии значительно больших, чем на других участках. Иначе говоря, необходимо управление нагружением на поверхность заготовки. это обеспечивает большие преимущества. В качестве примера рассмотрим процесс формообразования крыла автомобиля, приведенные в работе [6].

Сложная и разнообразная геометрия листовых деталей, особенности импульсного деформирования вызывают необходимость управления потоками энергии, подводимыми 
к заготовке или полуфабрикату на завершающих стадиях деформирования. Задача концентрации энергии в определенных зонах технологического объема возникла сразу после начала применения импульсных источников энергии в промышленном производстве. При использовании БВВ в качестве энергоносителя Ю. С. Навагиным было предложено с этой целью применить кумулятивные полости или полости с меньшей акустической жесткостью, чем у воды. Для управления временем выделения основной доли энергии применимы методы электрической задержки инициирования взрыва или детонирующие шнуры определенной длины, имеющие строго определенную скорость детонации.

При ЭГШ потребности пространственно-временного управления нагружением больше, а возможности такого управления шире. Рассмотрим подробнее потребности управления. В качестве примера возьмем типичную листовую деталь сложной формы типа «низкие коробки» - крыло легкового автомобиля (рис. 4).

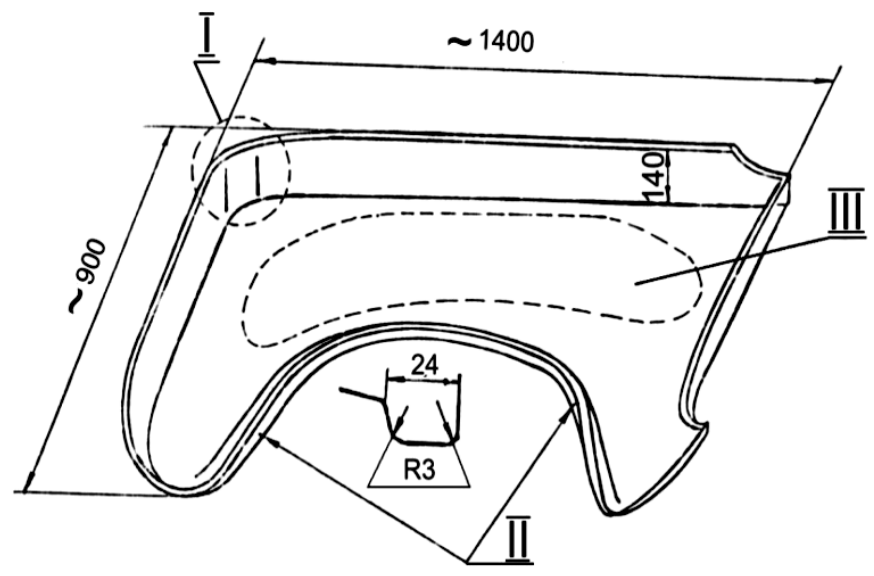

Рис. 4. Характерная крупногабаритная листовая деталь:

I, II, III - зоны детали, требующие при калибровке статического давления 90, 60 и 11 МПа соответственно

Представим ее как оболочку определенной генеральной формы с наложенными локальными элементами. Ее генеральная форма - коробка с относительно плоским дном и бортами. На эту форму наложены локальные элементы в виде рифта, криволинейного в плане (зона II), и донные сопряжения с радиусом кривизны, плавно изменяющимся вдоль борта. Наиболее резкое изменение кривизны этого элемента наблюдается в зоне I. Дно детали (зона III) имеет большой радиус кривизны, мало изменяющийся вдоль поверхности всей детали. Максимальная плотность потока энергии необходима для формообразования зоны I, значительно меньшие давления требуются для формообразования зоны II, а для формообразования зоны III требуются минимальные значения статического давления.

При калибровке детали по уравнению Лапласа для оформления заданной геометрии необходимо приложить давление 90 МПа. Если это давление приложить ко всей поверхности детали, то общее деформирующее усилие будет равно 75,5 MН. Однако для формообразования всей поверхности детали такого давления не требуется. Для формовки зон II и III необходимо соответственно 60 и 11 МПа. Таким образом, для изготовления детали общей площа-

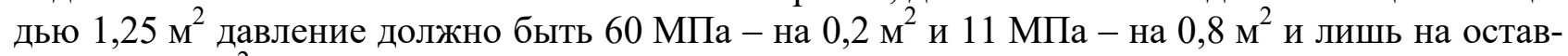
шиеся $0,25 \mathrm{~m}^{2}-90$ МПа. Это соответствует общему деформирующему усилию $26 \mathrm{MH}$ или почти в 3 раза меньше, чем при равномерно приложенном давлении. Таким образом, перераспределение нагрузки позволяет резко снизить потребные деформирующие усилия.

Анализ конструкции кузова украинского микроавтобуса «Сула» показывает, что необходимые усилия для штамповки кузовных деталей можно снизить в 2-16 раз, а энергозатраты - в 6-8 раз.

Квалиметрии оценить такое свойств техпроцесса пока возможно только экспертным методом. 
Вышеприведенный подход применим для оперативной оценки качества при подготовке производства (этап проектирования детали и технологии).

Таблица 1

Иерархическая совокупность на различных уровнях $i$-х свойств крупногабаритных листовых деталей

\begin{tabular}{|c|c|c|c|c|}
\hline \multicolumn{5}{|c|}{ Группы квалиметрических свойств продукции } \\
\hline$i=0$ & $i=1$ & $i=2$ & $i=3$ & $i=4$ \\
\hline 1 & 2 & 3 & 4 & 5 \\
\hline \multirow{23}{*}{ 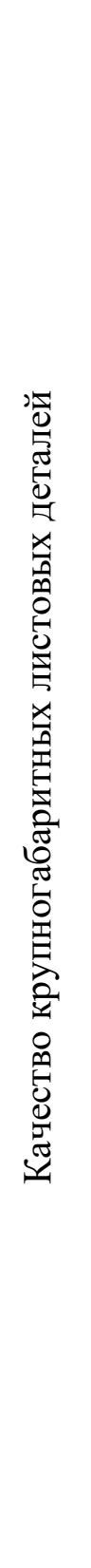 } & \multirow{3}{*}{ 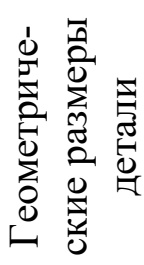 } & Габаритные формы & $\ldots$ & - \\
\hline & & $\begin{array}{c}\text { Соотношение отдель- } \\
\text { ных элементов }\end{array}$ & $\cdots$ & - \\
\hline & & Отклонение размеров & .. & - \\
\hline & \multirow{17}{*}{ 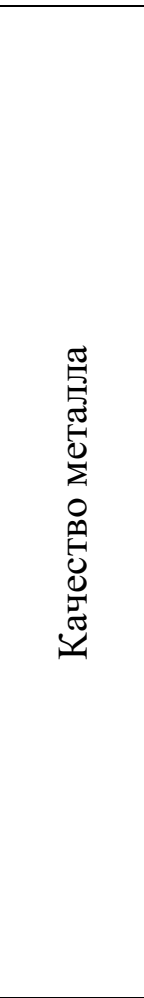 } & \multirow{2}{*}{ Структура } & Макроструктура & - \\
\hline & & & Микроструктура & - \\
\hline & & \multirow{10}{*}{ Механические свойства } & \multirow{4}{*}{ Растяжение } & $\begin{array}{l}\text { Временное } \\
\text { сопротивление }\end{array}$ \\
\hline & & & & Предел текучести \\
\hline & & & & Относительное удлинение \\
\hline & & & & Относительное сужение \\
\hline & & & \multirow[b]{2}{*}{ Ударная вязкость } & Работа разрушения \\
\hline & & & & $\begin{array}{l}\text { Процент вязкой } \\
\text { составляющей }\end{array}$ \\
\hline & & & \multirow{2}{*}{ Твердость } & $\ldots$ \\
\hline & & & & $\ldots$ \\
\hline & & & \multirow{2}{*}{$\begin{array}{c}\text { Технологическая } \\
\text { проба } \\
\end{array}$} & $\ldots$ \\
\hline & & & & $\ldots$ \\
\hline & & \multirow{3}{*}{ Внутренние дефекты } & $\ldots$ & - \\
\hline & & & $\ldots$ & - \\
\hline & & & Свариваемость & - \\
\hline & & \multirow[t]{2}{*}{ Специальные свойства } & $\begin{array}{c}\text { Коррозионная стой- } \\
\text { кость }\end{array}$ & $\cdots$ \\
\hline & & & $\ldots$ & - \\
\hline & \multirow{3}{*}{ 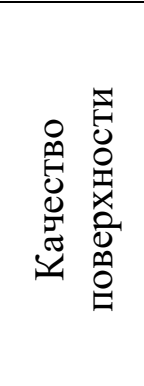 } & \multirow{3}{*}{$\begin{array}{c}\text { Состояние } \\
\text { поверхности, } \\
\text { образующей профиль }\end{array}$} & $\begin{array}{c}\text { Наличие или } \\
\text { отсутствие задиров } \\
(+;-) \\
\end{array}$ & $\cdots$ \\
\hline & & & $\begin{array}{c}\text { Неравномерность } \\
\text { вытяжки фланцев } \\
(+;-)\end{array}$ & $\cdots$ \\
\hline & & & $\begin{array}{l}\text { Наличие лимонной } \\
\text { корки }\end{array}$ & 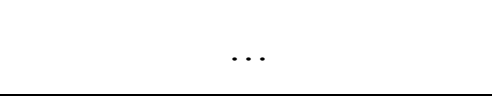 \\
\hline
\end{tabular}

Примечание. Прочерки обозначают отсутствие показателя. Многоточие - числовые значения

Общественный показатель качества на производственном этапе после её изготовления устанавливается с помощью таблиц иерархической совокупности на различных уровнях $i$-х свойств деталей, подвергнутых той или иной обработке. Численные значения этих свойств, измеренные (определенные) при контроле, приводятся к относительным значениям и свертываются в единый показатель. На первом этапе анализа рекомендуется пользоваться среднеарифметической функцией свертки. Пример используемого набора ПК показан в табл. 1. 


\section{ВЫВОДЫ}

1. Использование количественной оценки принимаемых решений при конструировании деталей, выборе способа их изготовления и действительного качества отштампованных деталей особенно важно для деталей большого габарита. Это определяет конкурентноспособность производства выпускаемой продукции.

2. Изложенный подход позволяет оценить квалиметрические показатели качества крупногабаритных листовых деталей. Для его использования в полной мере необходимы статистические данные штамповочных и инструментальных производств. Его эффективное применение желательно при использовании систем CAD/CAM/CAE/PDM и наличии среды INTERNET.

\section{СПИСОК ИСПОЛЬЗОВАННОЙ ЛИТЕРАТУРЫ}

1. Найзабеков А. В., Талмазон В. А., Шмидт Н. Ю. Квалиметрия в обработке металлов давлением. Алматы. РИК по УиМЛ, 2005. 134 с.

2. Ковтун І., Мазоренко Д. І., Пастухов В. І., Джолис П. А. Агрокваліметрія. Харків: РВП «Оригінал», $2000.314 \mathrm{c}$.

3. Бурдаков В. Д. Квалиметрия транспортных средств. Методика оценки эффективности использования. Москва: Изд-во стандартов, 1990. 166 с. $133 \mathrm{c}$.

4. Тараненко М. Е. Квалиметрия в листовой штамповке. Харьков: Нац. аэрокосм. ун-т «ХАИ», 2015.

5. Тараненко М. Е., Романцов А. В. Инжиниринг качества (техноквалиметрия). Нац. аэрокосм. ун-т «ХАИ», 2008. $129 \mathrm{c}$.

6. Тараненко М. Е. Электрогидравлическая штамповка: теорія, оборудование, техпроцессы. Нац. аэрокосм. ун-т «ХАИ», 2011. 272 с.

7. Михайлов А. Н. Функционально-ориентированные технологии машиностроения. Известия ТулГУ. Сер. Инструментальные и метрологические системы. Вып. 2. Труды Международной юбилейной научно-техн. конф. «Проблемы формообразования деталей при обработке резанием». Тула: ТулГУ, 2006. С. 24-32.

\section{REFERENCES}

1. Naizabekov A.V., Talmazon V.A., Schmidt N.Yu. Qualimetry in metal pressure processing. Almaty. RIK UiML. 2005. 134 p. (in Russian). (in Russian).

2. Kovtun I., Mazorenko D. I., Pastukhov V. I., Jolis P. A. Agrovalimetry. Xarkiv: RVP "Original". 2000.314 p.

3. Burdakov V.D. Vehicle qualimetry. Methodology for assessing the effectiveness of use. Moscow: Publishing house of standards. 1990. 166 p. (in Russian). (in Russian).

4. Taranenko M.E. Qualimetry in sheet stamping. Kharkov: Nat. Aerospace Un-ty "KhAI”. 2015.133 p.

5. Taranenko M.E., Romantsov A.V. Quality engineering (technoquality). Nat. Aerospace Un-ty "KhAI". 2008. 129 p. (in Russian).

6. Taranenko M.E. Electrohydraulic stamping: theory, equipment, technical processes. Nat. Aerospace. Un-ty "KhAI". 2011. 272 p. (in Russian).

7. Mikhailov A.N. Functional-oriented technologies of mechanical engineering. Bulletin of TulSU. Ser. Instrumental and metrological systems. Issue 2. Proceedings of the International Jubilee Scientific and Technical. conf. “Problems of forming parts during cutting”. Tula: TulSU, 2006, pp. 24-32. (in Russian).

Тараненко М. Е. - д-р техн. наук, профессор НАКУ «ХАИ».

НАКУ «ХАИ» - Национальный аэрокосмический университет им. М. Е. Жуковского «Харьковский авиационный институт», г. Харьков.

E-mail: $\underline{\text { m.taranenko@khai.edu https://orcid.org/0000-0003-4126-0979 }}$ 